Pacific Journal of Mathematics

EQUATIONAL DEFINABILITY OF ADDITION IN CERTAIN
RINGS HAL G. MOORE AND ADIT MOHAMED YAQUi 


\title{
EQUATIONAL DEFINABILITY OF ADDITION IN CERTAIN RINGS
}

\author{
Hal G. Moore and AdIL YaQub
}

Boolean rings and Boolean algebras, though historically and conceptually different, were shown by Stone to be equationally interdefinable. Indeed, in a Boolean ring, addition can be defined in terms of the ring multiplication and the successor operation (Boolean complementation) $x^{\wedge}=1+$ $x(=1-x)$. In this paper, it is shown that this type of equational definability of addition also holds in a much wider class of rings, namely periodic rings (ring satisfying $x^{m}=x^{n}$, $m \neq n)$ in which the idempotent elements are "well behaved." More generally, the following theorem is proved:

Suppose $R$ is a ring with unity 1 , not necessarily commutative. Suppose further that $R$ satisfies the identity $x^{n}=$ $x^{n+1} f(x)$ where $n$ is a fixed positive integer and $f(x)$ is a fixed polynomial with integer coefficients. If, further, the idempotent elements of $R$ commute with each other, then addition in $R$ is equationally definable in terms of multiplication in $R$ and the successor operation $x^{\wedge}=1+x$.

Some new classes of rings to which this theorem applies are exhibited.

1. The periodic case. In this section, we shall consider a periodic ring $R$ with unity 1 in which the idempotent elements commute with each other, and will give a direct proof of the equational definability of the " + " of $R$ in terms of " $X$ " and the successor operation $x^{\wedge}$. This direct proof avoids the axiom of choice. We begin with a formal definition of a periodic ring.

Definition 1. A ring $R$ is called periodic if there exist fixed integers $m$ and $n$ with $m>n \geqq 1$ such that for all $x$ in $R, x^{n}=x^{m}$.

LEMMA 1. Let $R$ be a periodic ring with unity 1. Then (i) For each $x$ in $R, x^{(m-n) n}$ is idempotent. (ii) $x$ is nilpotent if, and only if $x^{n}=0$.

Proof. (i) It can be shown by induction that the identity $x^{n}=x^{m}$ $(m>n \geqq 1)$ implies that for all positive integers $r$

$$
x^{n}=x^{n+r}\left(x^{m-n-1}\right)^{r} .
$$

In particular $x^{n}=x^{2 n}\left(x^{m-n-1}\right)^{n}$. Let $e=\left(x^{m-n}\right)^{n}$. It is readily verified that $e^{2}=e$, which proves (i). Part (ii) follows at once from equation (1). 
LEMMA 2. Let $R$ be a ring with unity 1 in which all of the idempotent elements commute with each other. Then all the idempotent elements of $R$ lie in the center of $R$.

Proof. Let $e^{2}=e \in R$. It is readily verified that for each $x \in R$, $e+e x-e x e$ is idempotent and hence $e(e+e x-e x e)=(e+e x-e x e) e$. Thus, $e x=e x e$. Similarly $x e=e x e$ and so $e x=x e$ proving the lemma.

To aid in our proof of the main theorem, we introduce some notation. Let $(R,+, \mathbf{X})$ be any (not necessarily periodic) ring with unity 1. Let $x \in R$. We define the (unary) successor operation $x^{\wedge}$ in $R$ by

$$
x^{\wedge}=x+1
$$

with an inverse successor operation $x^{\swarrow}$ given by

$$
x^{2}=x-1 \text {. }
$$

We also use following notation:

$$
x^{\wedge k}=\left(\cdots\left(\left(x^{\wedge}\right)^{\wedge}\right)^{\wedge} \cdots\right)^{\wedge}, \quad(k \text {-iterations }),
$$

with a similar definition for $x^{\vee k}$. Moreover for all $a, b \in R$ we define the (binary) operation

$$
a \times, b=\left(a^{\wedge} \times b^{\wedge}\right)^{`} \quad(=a+b+a b) .
$$

It is readily verified that for all $a \in R$,

$$
a \times \wedge=0 \times \wedge a=a .
$$

We are now in a position to give a direct proof of

THEOREM 1. Let $R$ be a periodic ring with unity 1 which satisfies the identity $x^{m}=x^{n}, m>n \geqq 1$. Suppose that all the idempotent elements of $R$ commute with each other. Then the "+" of $R$ is equationally definable in terms of the " $X$ " of $R$ and the successor operation `. Indeed for all $x, y \in R$ wo have

$$
\left\{\begin{array}{r}
x+y=\left[x\left(x^{m-n-1} y\right)^{\wedge}\left(x^{(m-n) n}\right)\right] \times \\
{\left[x^{\wedge}\left(\left(x^{\wedge}\right)^{m-n-1} y^{\vee}\right)^{\wedge}\left(\left(x^{(m-n) n}\right)^{`}\right)^{2}\right] .}
\end{array}\right.
$$

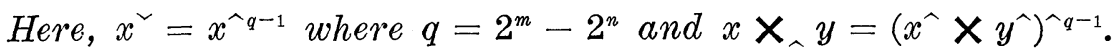

Proof. Let $x_{0}, y_{0}$ be arbitrary but fixed elements of $R$, and let

$$
e=x_{0}^{(m-n) n} \text {. }
$$


Then, by Lemmas $1,2, e$ is a central idempotent element of $R$. Let

$$
\operatorname{Re}=\{r e \mid r \in R\} ; \quad R(1-e)=\{r(1-e) \mid r \in R\} .
$$

The mapping

$$
\sigma: R \longrightarrow \operatorname{Re} \oplus R(1-e) ; \quad \sigma(r)=(r e, r(1-e)), \quad(r \in R),
$$

is readily seen to be an onto isomorphism:

$$
\sigma: R \cong \operatorname{Re} \oplus R(1-e), \quad(\sigma \text { is onto }) .
$$

Moreover, by (10),

$$
\sigma\left(x_{0}\right)=\left(x_{0} e, x_{0}(1-e)\right) ; \quad \sigma\left(y_{0}\right)=\left(y_{0} e, y_{0}(1-e)\right) .
$$

Now, since the operations in $\operatorname{Re} \oplus R(1-e)[=R]$ are componentwise, it suffices to verify (7) for both of the following substitutions [see (11), (12)]:

(i) $x=x_{0} e, y=y_{0} e$;

(ii) $x=x_{0}(1-e), y=y_{0}(1-e)$.

Verification of (7) when (i) holds:

In this case, $x_{0} e$ is a unit in Re, since by (8) and the fact that $e$ is a central idempotent,

$$
\left(x_{0} e\right)^{(m-n) n-1}\left(x_{0} e\right)=\left(x_{0} e\right)\left(x_{0} e\right)^{(m-n) n-1}=e e .
$$

Moreover, since $\left(x_{0} e\right)^{m}=\left(x_{0} e\right)^{n}$ and $x_{0} e$ is a unit in Re, we have

$$
\left(x_{0} e\right)^{m-n}=e e=e[=\text { identity of } \mathrm{Re}] \text {. }
$$

Hence the right side of (7), with $x=x_{0} e, y=y_{0} e$, reduces to [see (13)]

$$
\left[x_{0} e\left(e+\left(x_{0} e\right)^{m-n-1}\left(y_{0} e\right)\right)\right] \times, 0
$$

because $\left(\left(\left(x_{0} e\right)^{(m-n) n}\right)^{\smile}\right)^{2}=\left(e^{\smile}\right)^{2}=(e-e)^{2}=0$. Now, by (6) and (13), (14) reduces to

$$
x_{0} e+\left(x_{0} e\right)^{m-n}\left(y_{0} e\right)=x_{0} e+y_{0} e=x+y,
$$

which verifies (7) in this case.

Verification of (7) when (ii) holds:

To begin with, observe that $1-e$ is an idempotent element which is in the center of $R$, and in fact $1-e$ is the unity element of $R(1-e)$. Hence

$$
x^{(m-n) n}=\left[x_{0}(1-e)\right]^{(m-n) n}=x_{0}^{(m-n) n}(1-e)=e(1-e)=0,
$$

using (8). Thus $x=x_{0}(1-e)$ is a nilpotent element of $R(1-e)$, and hence (see $[3 ;$ p. 8]) 


$$
x^{\wedge}=(1-e)+x_{0}(1-e) \text { is a unit in } R(1-e) .
$$

Therefore, as in the above proof [see (13)],

$$
\begin{aligned}
\left(x^{\wedge}\right)^{m-n} & =\left[(1-e)+x_{0}(1-e)\right]^{m-n} \\
& =1-e[=\text { identity element of } R(1-e)] .
\end{aligned}
$$

Hence the right side of (7), with $x=x_{0}(1-e), y=y_{0}(1-e)$, reduces to [see (15)]

$$
0 \times{ }_{\wedge}\left[x^{\wedge}\left((1-e)+\left(x^{\wedge}\right)^{m-n-1} y^{\smile}\right)\left(0^{\smile}\right)^{2}\right]
$$

By (6), (16), (17), (3), the expression in (18) reduces to

$$
x^{\wedge}+\left(x^{\wedge}\right)^{m-n} y^{`}=x^{\wedge}+y^{\prec}=x+y,
$$

and again (7) is verified. Thus (7) is an identity of the ground ring $R$.

Now, since $x^{n}=x^{m}$ holds in $R$, in particular $\left(2^{m}-2^{n}\right) \cdot 1=0$. Let $q=2^{m}-2^{n}$. Then $x^{\wedge}=x$ in $R$ and thus $x^{\curlyvee}=x^{\wedge q-1}$. Therefore (5) implies that $x \times, y=\left(x^{\wedge} \times y^{\wedge}\right)^{\wedge-q}$ and the " + " of $R$ is indeed equationally definable via $(7)$ and these remarks in terms of the " $X$ " of $R$ and ${ }^{\wedge}$ only. This proves the theorem.

REMARK. Since a Boolean ring $R$ with identity has characteristic 2, $x^{\wedge}=x^{\curvearrowright}=x^{*}$ is the Boolean complement [5]. Also, a Boolean ring is periodic (satisfying $x=x^{2}$ ) and commutative [5]. Therefore $R$ satisfies all of the hypotheses of Theorem 1. Moreover, (7) now reduces to (since $m=2, n=1$ )

$$
x+y=\left[x y^{\wedge} x\right] \times \mathrm{X}_{\wedge}\left[x^{\wedge}\left(y^{\curlyvee}\right)^{\wedge}\left(x^{\curlyvee}\right)^{2}\right]=x y^{\wedge} \times x^{\wedge} y
$$

which becomes, using the definition of union [5] in a Boolean algebra,

$$
x+y=x y^{*} \cup x^{*} y \text {. }
$$

This is the familiar definition of addition in the Boolean case [5]. Therefore (7) may be viewed as a generalization to periodic rings of the formula for addition in the Boolean situation.

At the end of this paper we give some examples of rings, some commutative and some not commutative, for which Theorem 1 applies.

2. The general case. We now proceed to extend the results of the previous section to a class of rings satisfying certain types of polynomial identities. We begin with the following

Definition 2. A (not necessarily commutative) ring $R$ is called a local ring if each $x$ in $R$ is either nilpotent or else invertible in 
$R$. We are now prepared to prove the following

LeMma 3. Let $R$ be a ring with unity 1 , and let $n$ be a fixed positive integer. Suppose that $f(t)$ is a fixed polynomial with integer coefficients, and that, for all $x \in R$,

$$
x^{n}=x^{n+1} f(x) \text {. }
$$

$I f$, further, all the idempotent elements of $R$ commute with each other, then $R$ is isomorphic to a subdirect sum of local rings $R_{i}$ $(i \in \Gamma)$.

Proof. An easy induction, which we omit, shows that equation (19) implies

$$
x^{n}=x^{n+r}[f(x)]^{r} \text { for all positive integers } r .
$$

In particular $x^{n}=x^{2 n}[f(x)]^{n}$. Let $e=x^{n}[f(x)]^{n}$. Then $e^{2}=e$. Hence, by Lemma 2, we have the following:

$$
\text { If } x \in R \text {, then } e=x^{n}\{f(x)\}^{n} \text { is a central idempotent. }
$$

We recall, by Birkhoff's theorem [1], that $R$ is isomorphic to a subdirect sum of subdirectly irreducible rings $R_{i}(i \in \Gamma)$. We claim that each $R_{i}$ is a local ring. To prove this, let $\sigma_{i}: R \rightarrow R_{i}$ be the natural homomorphism of $R$ onto $R_{i}$. For $\bar{x} \in R_{i}$ let $x \in R$ be any preimage under $\sigma_{i}$. Let $e=x^{n}\{f(x)\}^{n}$. Then by (21), $e$ is a central idempotent in $R$. Let $\bar{e}=\sigma_{i}(e)$. Then $\bar{e}$ is an idempotent in the center of $R_{i}$. Now let

$$
I_{1}=\left\{\bar{e} \bar{r} \mid \bar{r} \in R_{i}\right\} ; \quad I_{2}=\left\{\bar{r}-\bar{e} \bar{r} \mid \bar{r} \in R_{i}\right\} .
$$

Since $\bar{e}$ is in the center of $R_{i}$, both $I_{1}$ and $I_{2}$ are ideals in $R_{i}$. Moreover $I_{1} \cap I_{2}=(\overline{0})$. But $R_{i}$ is subdirectly irreducible which forces either $I_{1}$ or $I_{2}$ to be $(\overline{0})$. If $I_{1}=(\overline{0}), \bar{e}=\bar{e}^{2}=\overline{0}$, hence $\bar{e}=\overline{0}$. If $I_{2}=(\overline{0})$, then $\bar{e}=\overline{1}$. Since $\bar{e}=(\bar{x})^{n}\{f(\bar{x})\}^{n}$, we have shown that

$$
\text { If } \bar{x} \in R_{i} \text {, then }(\bar{x})^{n}\{f(\bar{x})\}^{n}=\overline{0} \text { or }(\bar{x})^{n}\{f(\bar{x})\}^{n}=\overline{1} \text {. }
$$

Moreover, since $R_{i}$ clearly satisfies (20), we conclude from (22) that

$$
\text { If } \bar{x} \in R_{i} \text {, then }(\bar{x})^{n}=\overline{0} \text { or }(\bar{x})^{-1}=(\bar{x})^{n-1}\{f(\bar{x})\}^{n} \in R_{i} .
$$

Hence $R_{i}$ is a local ring, and the lemma is proved.

Next, we prove the following

LEMmA 4. In the notation and under all the hypotheses of Lemma 3 , the set $N_{i}$ of nilpotent elements in the local ring $R_{i}$ is 
an ideal in $R_{i}$ and $R_{i} / N_{i}$ is a field. Moreover, $N_{i}$ coincides with the Jacobson radical $J_{i}$ of $R_{i}$.

Proof. Suppose that $x \in J_{i}$. Then, as we proved in Lemma 3, $x^{n}\{f(x)\}^{n}$ is an idempotent element in $J_{i}$, and hence $x^{n}\{f(x)\}^{n}=0$. Therefore, by (20), $x^{n}=0$, and hence $J_{i} \subseteq N_{i}$. Now, suppose that $a \in N_{i}$ and $x \in R_{i}$. Since $R_{i}$ is a local ring, $a x$ is either nilpotent or is a unit in $R_{i}$. It is easy to see that $a x$ is not a unit in $R_{i}$ (since $a$ is nilpotent), and hence $a x$ is nilpotent. Therefore, $a x$ is right quasi-regular for all $x$ in $R_{i}$, and hence $a \in J_{i}$. Thus, $N_{i} \subseteq J_{i}$, and hence $N_{i}=J_{i}$. Now, observe that the identity $x^{n}=x^{n+1} f(x)$ is inherited by the division ring $R_{i} / N_{i}$ and, moreover, both $n$ and $f(x)$ are fixed. Hence, $R_{i} / N_{i}$ is a field, and the lemma is proved.

LEMMA 5. In the notation and under all the hypotheses of Lemmas 3 and 4 , there exists a monic polynomial $g(x)$ with integer coeficients and an integer $m>1$ such that for all $x$ in $R$,

$$
x^{m}=x^{m+1} g(x) \text {. }
$$

Proof. Let $x=2$ in (19). This gives $2^{n}=2^{n+1} f(2)$, and hence the characteristic of $R$ is a positive integer $q$. Now let $p_{1}, p_{2}, \cdots, p_{s}$ be all the distinct prime factors of $q$. Let $x \in R_{i}, x \notin N_{i}$, and let $\sigma_{i}: R_{i} \rightarrow R_{i} / N_{i}$ be the natural homomorphism of $R_{i}$ onto the field $R_{i} / N_{i}$. Let $\sigma_{i}: x \rightarrow \bar{x}$. Note that the field $R_{i} / N_{i}$ has prime characteristic, and moreover the subring $\langle\bar{x}\rangle$ generated by $\bar{x}$ is a finite field; that is,

$$
\langle\bar{x}\rangle=G F\left(p_{j}^{k} j\right) \subseteq R_{i} / N_{i} .
$$

Moreover, since the characteristic of the field $R_{i} / N_{i}$ must divide the characteristic $q$ of $R$, it follows that the prime $p_{j}$ is one of the prime factors of $q$. Also in view of (19) we have

$$
(\bar{x})^{n}=(\bar{x})^{n+1} f(\bar{x})
$$

for all $\bar{x} \in R_{i} / N_{i}$. We define the polynomial $h(t)$ of degree $a$ by

$$
h(t)=t^{n+1} f(t)-t^{n} .
$$

Since $f(t)$ has integer coefficients, so does $h(t)$. From (25) we conclude that $k_{j}$ is the degree of the irreducible (minimal) polynomial which $\bar{x}$ satisfies over $G F\left(p_{j}\right)$ and hence $k_{j} \leqq a$, where we now view $h(t)$ as a polynomial in $G F\left(p_{j}\right)[t]$. But then $k_{j}$ divides $a$ ! and, hence,

$$
t^{p_{j}^{k} j}-t \text { divides } t^{p_{j}^{a !}}-t \text {. }
$$


We can, therefore, conclude from (25) and (26) that

$$
(\bar{x})^{p_{j}^{a !}}-\bar{x}=\overline{0} \quad\left(=\text { the zero of } R_{i} / N_{i}\right) \text {. }
$$

Therefore, for $x \in R_{i}, x^{p_{j}^{a}}-x$ is nilpotent in $R_{i}$. Since, moreover, $R_{i}$ satisfies (20), we conclude that

$$
\left(x^{p_{j}^{a !}}-x\right)^{n}=0 \text { for all } x \in R_{i} .
$$

(Note that if $x$ is in $N_{i}$, then $x^{n}=0$, from which (27) also follows.) Now define the monic polynomial $u(x)$ by

$$
u(x)=\left[\prod_{j=1}^{s}\left(x^{p_{j}^{a !}}-x\right)^{2 n}\right](x-1)
$$

where, of course $p_{1}, p_{2}, \cdots, p_{s}$ are the distinct prime factors of $q$. Observe that the coefficient of the lowest degree term in $u(x)$ is -1 . Moreover, by (27), if $x$ is any element of any of the rings $R_{i}(i \in \Gamma)$, $u(x)=0$. Hence, in view of the fact that the operations of a subdirect sum are componentwise, $u(x)=0$ for all $x$ in the ground ring $R$. Thus, by (28) we see that, for some integer $m>1$ and some monic polynomial $g(x)$ with integer coefficients equation (24) holds, and the lemma is proved.

LEMMA 6. Suppose that $R$ is a ring of positive characteristic $q$ and with unity element. Then,

$$
x^{\swarrow}=x^{\wedge q-1} \text { and } x \times, y=\left(x^{\wedge} \times y^{\wedge}\right)^{\wedge q-1} .
$$

Moreover, any monic polynomial $f(x)$ with integer coefficients and zero constant term is expressible in terms of the operations $X$ and in $R$.

Proof. To avoid any possible confusion, let us denote the unity of $R$ by $e$. Then $x^{\wedge}=x+e$ and $x^{\curvearrowright}=x-e$. Hence, (see (4)),

$$
x^{\wedge} q-1=x+(q-1) e=x-e=x^{`} .
$$

Now recalling the definition of $X_{\wedge}$ in (5) we have

$$
x \times{ }_{\wedge} y=\left(x^{\wedge} \times y^{\wedge}\right)^{\vee}=\left(x^{\wedge} \times y^{\wedge}\right)^{\wedge q-1}
$$

which proves (29).

To complete the proof of the lemma, consider the monic polynomial

$$
f(t)=t^{n}+a_{1} t^{n-1}+a_{2} t^{n-2}+\cdots+a_{n-1} t
$$

with integer coefficients and zero constant term. Since by hypothesis 
the characteristic of $R$ is $q, q x=0$ for all $x \in R$. Thus we may assume, without loss of generality, that each of the integers $a_{i}$ $(i=1,2, \cdots, n-1)$ in (30) is positive. Then, by (4), we see that

$$
f(x)=x\left(\cdots\left(x\left(x\left(x\left(x^{\wedge a_{1}}\right)\right)^{\wedge a_{2}}\right)^{\wedge a_{3}}\right) \cdots\right)^{\wedge a_{n-1}} .
$$

This finishes the proof of the lemma.

With the aid of these lemmas we are now in a position to prove the following main theorem.

THEOREM 2. Let $R$ be a ring with unity 1 ; let $n$ be a fixed positive integer, and let $f(t)$ be a fixed polynomial with integer coefficients such that for all $x \in R$

$$
x^{n}=x^{n+1} f(x) \text {. }
$$

If, further, all the idempotent elements of $R$ commute with each other, then the "+" of $R$ is equationally definable in terms of the " $X$ " of $R$ and the (unary) successor operation ".

Proof. By Lemma 5 there exists a monic polynomial $g(t)$ with integer coefficients and an integer $m>1$ such that $x^{m}=x^{m+1} g(x)$ for all $x \in R$. We claim that $R$ satisfies the following identity:

$$
\left\{\begin{array}{c}
x+y=\left[x\left(x^{m-1}(g(x))^{m} y\right)^{\wedge}(x g(x))^{m}\right] \times \text {, } \\
\quad\left[x^{\wedge}\left(\left(x^{\wedge}\right)^{m-1}\left(g\left(x^{\wedge}\right)\right)^{m} y^{\llcorner}\right)^{\wedge}\left(\left((x g(x))^{m}\right)^{`}\right)^{2}\right] .
\end{array}\right.
$$

To prove this we recall first that by Lemma $3, R$ is isomorphic to a subdirect sum of local rings $R_{i}(i \in \Gamma)$. Since the operations in a subdirect sum are componentwise it suffices to verify (32) for each local ring $R_{i}$. To this end we distinguish two cases:

Case 1. $x$ is a unit in $R_{i}$. Note that $x^{m}=x^{m+1} g(x)$ holds in $R_{i}$ and hence $x g(x)=1$. Therefore the right side of (32) reduces to (see (3))

$$
\left[x\left(1+x^{m-1}(g(x))^{m} y\right)\right] \times \mathbf{X}_{\wedge} 0,
$$

since $\left(\left((x g(x))^{m}\right)^{`}\right)^{2}=\left(1^{`}\right)^{2}=0^{2}=0$. But then the right side (see (6)) reduces to

$$
x+x^{m}(g(x))^{m} y=x+(x g(x))^{m} y=x+y
$$

as desired.

Case 2. $x$ is a nilpotent element in $R_{i}$-the only other possibility in a local ring. In this case $1+x=x^{\wedge}$ is a unit in $R_{i}$. Therefore, as in Case 1, we have $x^{\wedge} g\left(x^{\wedge}\right)=1$. Moreover, since $x^{m}=x^{m+1} g(x)$, 
by reiterating we get

$$
x^{m}=x^{m+r}(g(x))^{r}
$$

for all positive integers $r$. Since $x$ is nilpotent, (33) readily implies that $x^{m}=0$, and, therefore, $(x g(x))^{m}=0$. Thus, the right side of (32) reduces to

$$
\begin{aligned}
0 \times{ }_{\wedge} & {\left[x^{\wedge}\left(1+\left(x^{\wedge}\right)^{m-1}\left(g\left(x^{\wedge}\right)\right)^{m} y^{`}\right)\left(0^{`}\right)^{2}\right] } \\
& =\left(x^{\wedge}+\left(x^{\wedge}\right)^{m}\left(g\left(x^{\wedge}\right)\right)^{m} y^{`}\right)(-1)^{2} \\
& =x^{\wedge}+\left(x^{\wedge} g\left(x^{\wedge}\right)\right)^{m} y^{`}=x^{\wedge}+y^{`} \\
& =x+y .
\end{aligned}
$$

These two cases demonstrate that (32) is an identity satisfied by all elements $x$ and $y$ of each local ring $R_{i}(i \in \Gamma)$. Therefore, (32) is an identity of the ground ring $R$.

To complete the proof, we first observe that by setting $x=2$ in (31), we get

$$
2^{n}=2^{n+1} f(2) \text {. }
$$

Thus, the characteristic of $R$ is a positive integer $q$. Hence, by (2)-(5),

$$
x^{\curlyvee}=x^{\wedge q-1} \text { and } x \times, y=\left(x^{\wedge} \times y^{\wedge}\right)^{\wedge q-1}
$$

Now let

$$
h(t)=t^{m-1}(g(t))^{m}
$$

Note that since $g(t)$ is a monic polynomial with integer coefficients and $m>1, h(t)$ is also a monic polynomial with integer coefficients whose constant term is zero. Therefore, by Lemma $6, h(t)$ is expressible as a primitive composition of the operations $X$ and $\uparrow$, say

$$
h(t)=\Phi\left(t ; \times,^{\wedge}\right) \text {. }
$$

By (35) and (36), it follows that

$$
x^{m-1}(g(x))^{m}=\Phi\left(x ; \mathbf{X},{ }^{\wedge}\right) \text { and }\left(x^{\wedge}\right)^{m-1}\left(g\left(x^{\wedge}\right)\right)^{m}=\Phi\left(x^{\wedge} ; \mathbf{X},{ }^{\wedge}\right)
$$

Also by Lemma $6, x g(x)$ is expressible as a primitive composition of the operations $X$ and $\hat{\text {, say }}$

$$
x g(x)=\psi\left(x ; \times,{ }^{\wedge}\right) .
$$

In view of (34), (37), and (38), the right side of the identity (32) is expressible in terms of the two operations $X$, and $\hat{\wedge}$, which proves the theorem. 
3. Examples. We turn now to some examples of rings, some commutative and some not commutative, to which our theorems apply.

EXAMPLE 1. Let $R$ be any Boolean ring with unity [5]; more generally, let $R$ by any $p$-ring with unity; i.e., $R$ satisfies $x^{p}=x, p x=0$, $p=$ prime. (See [4], [2], and [7].) Then the "+" of $R$ is equationally definable in terms of " $X$ " and "^".

EXAMPLE 2. Let $R$ be any ring with unity in which, for a fixed $n>1$ and every $x \in R, x^{n}=x$. Then here too the " + " of $R$ is equationally definable in terms of " $X$ " and " $"$ ".

It should be pointed out that the rings of Examples 1 and 2 are necessarily commutative, (see [3]; p. 217), as are, of course the rings of the next example.

EXAMPLE 3. Let $R$ be the ring $Z_{n}$ of integers modulo $n$. It can be shown that $R$ is periodic; in fact, $R$ satisfies the identity

$$
x^{n \varphi(n)}=x^{2 n \varphi(n)}
$$

where $\varphi(n)$ is the familiar Euler $\varphi$-function. Therefore, by Theorem 1 , the " + " of $R$ is equationally definable in terms of " $X$ " and . Indeed equation (7) now becomes

$$
\begin{aligned}
x+y= & {\left[x\left(x^{n \varphi(n)-1} y\right)^{\wedge}\left(x^{(n \varphi(n))^{2}}\right)\right] \times \widehat{\times} } \\
& {\left[x^{\wedge}\left(\left(x^{\wedge}\right)^{n \varphi(n)-1} y^{\curlyvee}\right)^{\wedge}\left(\left(x^{(n \varphi(n))^{2}}\right)^{\curlyvee}\right)^{2}\right] . }
\end{aligned}
$$

This formula for " + " is much simpler than that given in [6].

The next two examples demonstrate that our theorems also apply to some rings which are not commutative.

EXAMPLE 4. Let $F=G F\left(p^{k}\right)$ be a finite field and let $R$ be the ring of those $n \times n$ upper triangular matrices over $F$ in which all of the entries on the main diagonal are equal. It can be shown that $R$ is a finite local ring whose only idempotent elements are the zero matrix and the identity matrix. The ring $R$ is also a periodic ring; in fact, $R$ satisfies the following identity:

$$
x^{p^{n k}}=x^{p^{(n+1) k}} \text {. }
$$

Therefore $R$ satisfies all of the hypotheses of Theorem 1; and hence, the "+" of $R$ is equationally definable in terms of " $X$ " and ". Observe that $R$ is not commutative for $n>2$ even over $G F(2)$.

Example 4 can be generalized as follows. 
Example 5. Let $R$ be any finite (not necessarily commutative) ring in which all of the idempotent elements commute with each other. Then the " + " of $R$ is equationally definable in terms of " $X$ " and $\uparrow$.

To prove this we let $R=\left\{x_{1}, x_{2}, \cdots, x_{k}\right\}$. Now for any $x_{i} \in R$ let

$$
S=\left\{x_{i}, x_{i}^{2}, \cdots, x_{i}^{k+1}\right\} .
$$

Since $S$ contains $k+1$ elements of $R$, there must exist integers $r_{i}$ and $s_{i}$ such that $1 \leqq r_{i}<s_{i} \leqq k+1$ for which

$$
x_{i}^{r_{i}}=x_{i}^{s_{i}} \text {. }
$$

Therefore, as in the proof of Lemma 1(i), it must follow that $x_{i}^{\left(s_{i}-r_{i}\right) r_{i}}$ is idempotent. Now let

$$
n=\prod_{i=1}^{k}\left(s_{i}-r_{i}\right) r_{i} \text { and } m=2 n .
$$

Then we see that each $x \in R, x^{m}=x^{n}$. Hence, $R$ is periodic. Therefore, again by Theorem 1 , the "+" of $R$ is equationally definable in terms of the " $X$ " of $R$ and the successor operation .

The rings in Examples 4 and 5 are the first known examples of rings in which the commutative operation of ring addition is equationally definable in terms of a not commutative ring multiplication and a successor operation ^. One might ask just how noncommutative a ring may be for this to still be possible.

In conclusion, we would like to express our indebtedness and gratitude to the referee for his helpful suggestions and valuable comments.

\section{REFERENCES}

1. G. Birkhoff, Subdirect unions in universal algebras, Bull. Amer. Math. Soc., 50 (1944), 764-768.

2. A. L. Foster, p-rings and ring-logics, University of California Publ., 1 (1951), 385-396.

3. N. Jacobson, Structure of rings, Amer. Math. Colloq. Publ., 37 (1964).

4. N. H. McCoy and D. Montgomery, A representation of generalized Boolean rings, Duke Math. J., 3 (1937), 455-459.

5. M. H. Stone, The theory of representations of Boolean algebras, Trans. Amer. Math. Soc., 40 (1936), 37-111.

6. A. Yaqub, On the theory of ring-logics, Canad. J. Math., 8 (1956), 323-328.

7. - On certain finite rings and ring-logics, Pacific J. Math., 12 (1962), 785-790.

Received February 16, 1977 and in revised form August 15, 1977.

Brigham Young University at Provo, UT 84112

AND

University of California at Santa Barbara, CA 93106 



\section{PACIFIC JOURNAL OF MATHEMATICS}

\section{EDITORS}

RICHARD ARENS (Managing Editor)

University of California

Los Angeles, CA 90024

Charles W. Curtis

University of Oregon

Eugene, OR 97403

C. C. Moore

University of California

Berkeley, CA 94720

\section{J. DugundJI}

Department of Mathematics

University of Southern California

Los Angeles, CA 90007

R. FInN and J. Milgram

Stanford University

Stanford, CA 94305

\section{ASSOCIATE EDITORS}
E. F. BECKENBACH
B. H. NeumanN
F. WOLF
K. YoSHIDA

\section{SUPPORTING INSTITUTIONS}

\author{
UNIVERSITY OF BRITISH COLUMBIA \\ CALIFORNIA INSTITUTE OF TECHNOLOGY \\ UNIVERSITY OF CALIFORNIA \\ MONTANA STATE UNIVERSITY \\ UNIVERSITY OF NEVADA, RENO \\ NEW MEXICO STATE UNIVERSITY \\ OREGON STATE UNIVERSITY \\ UNIVERSITY OF OREGON
}

\author{
UNIVERSITY OF SOUTHERN CALIFORNIA \\ STANFORD UNIVERSITY \\ UNIVERSITY OF HAWAII \\ UNIVERSITY OF TOKYO \\ UNIVERSITY OF UTAH \\ WASHINGTON STATE UNIVERSITY \\ UNIVERSITY OF WASHINGTON
}

The Supporting Institutions listed above contribute to the cost of publication of this Journal, but they are not owners or publishers and have no responsibility for its content or policies.

Mathematical papers intended for publication in the Pacific Journal of Mathematics should be in typed form or offset-reproduced, (not dittoed), double spaced with large margins. Please do not use built up fractions in the text of the manuscript. However, you may use them in the displayed equations. Underline Greek letters in red, German in green, and script in blue. The first paragraph or two must be capable of being used separately as a synopsis of the entire paper. Items of the bibliography should not be cited there unless absolutely necessary, in which case they must be identified by author and journal, rather than by item number. Manuscripts, in triplicate, may be sent to any one of the editors. Please classify according to the scheme of Math. Reviews, Index to Vol. 39. All other communications should be addressed to the managing editor, or Elaine Barth, University of California, Los Angeles, California, 90024.

50 reprints to each author are provided free for each article, only if page charges have been substantially paid. Additional copies may be obtained at cost in multiples of 50 .

The Pacific Journal of Mathematics is issued monthly as of January 1966. Regular subscription rate: $\$ 72.00$ a year (6 Vols., 12 issues). Special rate: $\$ 36.00$ a year to individual members of supporting institutions.

Subscriptions, orders for numbers issued in the last three calendar years, and changes of address should be sent to Pacific Journal of Mathematics, 103 Highland Boulevard, Berkeley, California, 94708. Older back numbers obtainable from Kraus Periodicals Co., Route 100, Millwood, NY 10546.

PUBLISHED BY PACIFIC JOURNAL OF MATHEMATICS, A NON-PROFIT CORPORATION

Printed at Kokusai Bunken Insatsusha (International Academic Printing Co., Ltd.). 8-8, 3-chome, Takadanobaba, Shinjuku-ku, Tokyo 160, Japan.

Copyright (C) 1978 by Pacific Journal of Mathematics

Manufactured and first issued in Japan 


\section{Pacific Journal of Mathematics

Vol. 74, No. $2 \quad$ June, 1978

Aharon Atzmon, Spectral synthesis in some spaces of bounded continuous

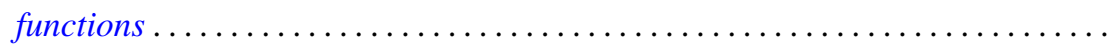

Karl Egil Aubert and Isidor Fleischer, Tensor products of ideal systems and their modules.............................................

Richard F. Basener, Several dimensional properties of the spectrum of a uniform

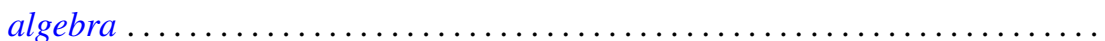

R. H. Bing and Michael Peter Starbird, Super triangulations ............. 307

Andrew Carson, Coherent polynomial rings over regular rings of finite index .....

Robert M. DeVos and Frederick W. Hartmann, Sequences of bounded summability domains .................................................

George Grätzer and R. Padmanabhan, Symmetric difference in abelian groups ....

Robert L. Griess, Jr., A remark about groups of characteristic 2-type and

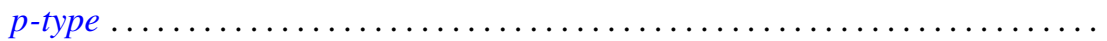

Emil Grosswald and F. J. Schnitzer, A class of modified $\zeta$ and L-functions........

Jutta Hausen and Johnny Albert Johnson, Ideals and radicals of some

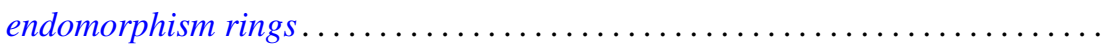

Jean Ann Larson, A solution for scattered order types of a problem of Hagendorf ............................................

Peter A. McCoy, Extremal properties of real biaxially symmetric potentials in $E^{2(\alpha+\beta+2)}$

Héctor Alfredo Merklen, Hereditary crossed product orders .

Hal G. Moore and Adil Mohamed Yaqub, Equational definability of addition in certain rings...

Robert Laurens Moore, Reductivity in $C^{*}$-algebras and essentially reductive operators. . .

Joseph Alvin Neisendorfer, Lie algebras, coalgebras and rational homotopy theory for nilpotent spaces...

William Raymond Nico, Bounded monoids

Richard Paul Osborne, Simplifying spines of 3-manifolds ...

Richard Paul Osborne, The simplest closed 3-manifolds. With an appendix by Osborne and J. Yelle.

Clayton Collier Sherman, The $K$-theory of an equicharacteristic discrete valuation ring injects into the $K$-theory of its field of quotients.... .

Mitchell Herbert Taibleson, The failure of even conjugate characterizations of $H^{1}$

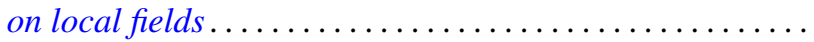

Keti Tenenblat, On characteristic hypersurfaces of submanifolds in Euclidean space ...................................

Jeffrey L. Tollefson, Involutions of Seifert fiber spaces..........

Joel Larry Weiner, An inequality involving the length, curvature, and torsions of a curve in Euclidean $n$-space .......................

Neyamat Zaheer, On generalized polars of the product of abstract homogeneous polynomials.... 